\title{
Two New Records of the Ichneumonidae (Hymenoptera) Species from Korea
}

\author{
Chang-Jun Kim, Gyu-Won Kang, Jong-Wook Lee* \\ Department of Life Sciences, Yeungnam University, Gyeongsan 712-749, Korea
}

\begin{abstract}
Previously, 13 species in six genera of the subfamily Banchinae and 29 speices in 16 genera of the subfamily Pimplinae were known from Korea. In this paper, two species, Rynchobanchus minomensis (Uchida, 1933) and Theronia laevigata nigra Uchida, 1928 are new to Korea. Also, the genus Rynchobanchus is recorded for the first time in Korea. Only one species of Theronia, Theronia atalantae (Poda, 1761), was recorded from Korea by Uchida (1928). New Korean names proposed for these two species are "Eol-gul-ga-si-maep-si-beol" and "Geom-jeong-nap-jak-maep-si-beol" for the $R$. minomensis and T. laevigata nigra, respectively. A key to the species of Theronia, diagnosis and photographs of diagnostic characters of them are provided.
\end{abstract}

Keywords: Rynchobanchus, Theronia, Banchinae, Pimplinae, Korea

\section{INTRODUCTION}

The subfamilies, Banchinae Wesmael, 1845 and Pimplinae Wesmael, 1845 are large-sized groups belonging to the Family Ichneumnidae. The Banchinae includes 1,758 species of 65 genera and Pimplinae includes 1,686 species of 78 genera around the world (Yu et al., 2012).

The Genus Rynchobanchus is a small genus with six species in the tribe Banchini (Hymenoptera: Ichneumonidae: Banchinae): R. bicolor Kriechbaumer, 1894; R. flavopictus Heinrich, 1937; R. maculicornis Sheng, Liu \& Wang 1995; $R$. minomensis (Uchida, 1933); R. niger Sheng, Li \& Pang 1997; R. nigriventris Meyer, 1927 (Yu et al., 2012). Of these, only the species $R$. minomensis is known to distribute in the Eastern Palaearctic region (China, Japan and Russia), but of which any specimen has not been found in Korea despite of possibility of its Korean occurrence. The genus Rynchobanchus can be easily distinguished from other genera of Banchini by the following combination of characteristics: 1) prepectal carina absent; 2) apical transverse carina of propodeum absent; 3) pleural carina of propodeum absent; 4) apex of scutellum rounded.

The another genus treated herein Theronia is a moderatesized group belonging to the tribe Pimplini (Hymenoptera: Ichneumonidae: Pimplinae) consisting of 39 species. Only one species of this genus, Theronia atalantae (Poda, 1761), was recorded from Korea by Uchida (1928). The biology of this genus has been studied well, and most species are specialized endoparasitoid or hyperparasitoid of the Lepidopteran species (Yu et al., 2012). The genus Theronia can be easily distinguished from other genera of Pimplini by the following combination of characteristics: 1) epicnemial carina present; 2) mesopleural suture centrally angled; 3) propodeum with strong carinae, and spiracles elongately oval; 4) forewing with 3r-m, enclosing a rhombic areolet.

In the present study two unrecorded species, Rynchobanchus minomensis (Uchida, 1933) and Theronia laevigata nigra Uchida, 1928 is reported including a key to the Korean species of Theronia.

The morphological terminologies follow those of Townes (1969) and Yu et al. (2012). Photographs were taken using an AxioCam MRc5 camera attached to a stereomicroscope (Stemi SV 11 Apo; Carl Zeiss, Göttingen, Germany), processed using AxioVision40AC software (Carl Zeiss), and optimized with an i-delta imaging system (i-Delta 2.6; iMTechnology, Daejeon, Korea).

All specimens used were deposited at the Yeungnam University, South Korea (YNUE). Abbreviations for the type information, collecting method and the province of collection localities are as follows: TL, type locality; TS, type spe-

\footnotetext{
(C) This is an Open Access article distributed under the terms of the Creative Commons Attribution Non-Commercial License (http://creativecommons.org/ licenses/by-nc/3.0/) which permits unrestricted non-commercial use, distribution, and reproduction in any medium, provided the original work is properly cited.

*To whom correspondence should be addressed

Tel: 82-53-810-2376, Fax: 82-53-811-2376

E-mail: jwlee1@ynu.ac.kr 
cies; MT, malaise trap; CB, Chungcheongbuk-do; CN, Chungcheongnam-do; DG, Daegu-si; DJ, Daejeon-si; GB, Gyeongsangbuk-do; GG, Gyeonggi-do; GJ, Gwangju-si; GN, Gyeongsangnam-do; GW, Gangwon-do; JB, Jeollabuk-do; JJ, Jejudo; JN, Jeollanam-do; SU, Seoul-si; US, Ulsan-si.

\section{SYSTEMATIC ACCOUNTS}

Order Hymenoptera

Family Ichneumonidae Latreille, 1802

Subfamily Banchinae Wesmael, 1845

1*Genus Rynchobanchus Kriechbaumer, 1894

Rhynchobanchus Kriechbaumer, 1894: 59. TS: Rhynchobanchus bicolor Kriechbaumer.
Acrogonia Kriechbaumer, 1896: 369. TS: Acrogonia semirufa Kriechbaumer.

Acrogoniella Schulz, 1911: 23.

2*Rynchobanchus minomensis (Uchida, 1933) (Fig. 1)

Exetastes minomensis Uchida, 1933: 74. Type: 우, TL: Mino in Osaka (Sapporo, Japan).

Rhynchobanchus minomensis Townes, Momoi \& Townes, 1965: 235.

Material examined. Korea: $1 \sigma^{7}, \mathrm{CB}$ : Cheondong-ri, Mt. Sobaek, 12 May 1994, Kim JG; 2 『 §, Dannyang-gun, Cheondong-ri, 11 May 1994, Cho OH; $1 \sigma^{\nearrow}$, ditto, 14 May 1994, Park HY; 1 ð, Dannyang-gun, Dannyang-eup, Mt. Sobaek, 11 May 1994, Kim TD; 1 우2 $\sigma^{\nearrow}$ 『, ditto, 23 May 1997, Lee JW; 3 ๙ $^{\nearrow}$, ditto, 12 May 1994, Jang JH; 4 ఠ ठ ,

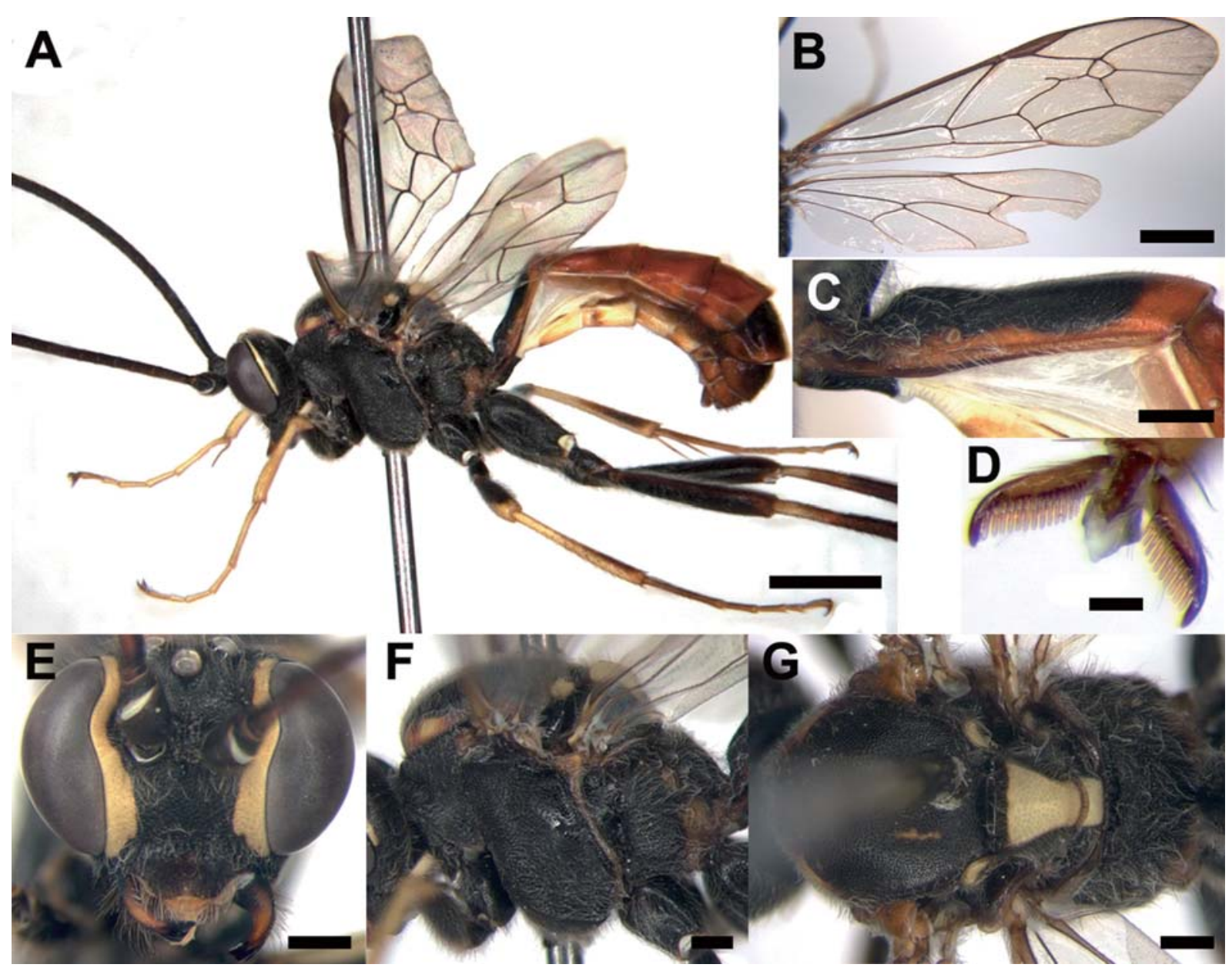

Fig. 1. Rynchobanchus minomensis (Uchida, 1933), female. A, Lateral habitus; $B$, Fore wing; C, Petiole in lateral; $D$, Tarsal claw; $E$, Head in anterior; $F$, Mesosoma in lateral; $G$, Mesosoma in dorsal. Scale bars: $A, B=2 \mathrm{~mm}, C, E-G=0.5 \mathrm{~mm}, D=0.1 \mathrm{~mm}$.

Korean name: ${ }^{1 *}$ 얼굴가시맵시벌속(신칭), ${ }^{2 *}$ 얼굴가시맵시벌 (신칭) 
ditto, Kim JI; $2 \sigma^{\nearrow} \sigma^{\nearrow}$, ditto, 13 May 1994, Lee MK; $28 \sigma^{\nearrow} \sigma^{\nearrow}$, ditto, 12 May 1994, ?; $3 \sigma^{\nearrow}$ ð, ditto, Choi AJ; $3 \sigma^{\nearrow} \sigma^{\nearrow, ~ d i t t o, ~}$ Jang JH; $3 \sigma^{\nearrow} \sigma^{\nearrow}$, ditto, Kim JI; $4 \sigma^{\nearrow} \sigma^{\nearrow}$, ditto, Seo MJ; 2 우 우, ditto, Lee MK; $3 \sigma^{\nearrow} \sigma^{\nearrow}$, ditto, Kim TS; $3 \sigma^{\nearrow} \sigma^{\nearrow}$, Goesan-gun, Cheongcheon-myeon, Mureung-ri, 22 Apr 2006, Oh SH;

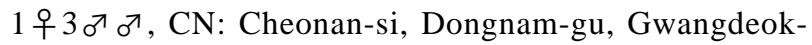
myeon, Gwangdeok-ri, 22 Apr 1984, Lee JW; 1 § , Gongjusi, Gyeryong-myeon, Jungjang-ri, Mt. Gyeryong, Gapsa, 24 Apr 1983, Park HC; 1 ð , DG: Dalseo-gu, Songhyeon-dong, Mt. Daedeok, 11 May 1992, Kim KM; 3 ठ ð , DJ: Dong-gu, Secheon-dong, 20 May 1996, No MS; 1 우, GW: Hongcheongun, Nae-myeon, Gachilbong, 24 May 1996, Kim HY; 1 우, ditto, 25 May 1996, Lee KH; $29 \sigma^{\nearrow}$ ๙, Taebaek-si, Hyeoldong, Mt. Taebaek, 13 May 1991, Lee JW; 30 ఠ ఠ , Taebaek-si, Mungoksodo-dong, Danggol, 6 May 1999, Lee JW; 1우, GG: Gapyeong-gun, Buk-myeon, Mt. Myeongji, 21

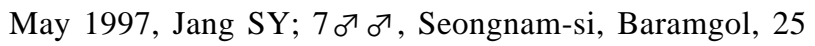
Jun 1984, Lee JW; 2 우 우, Gwacheon-si, Galhyeon-dong, 126-5, 17 Apr 1983, Lee JW; 1 우, GB: Cheongdo-gun, Unmun-myeon, Sinwon-ri, Mt. Unmun, 24 Aug 2008, Lee JW; 5 ð ア , Gyeongsan-si, Dae-dong, Yeungnam University, 27 Apr 1996, Kwon OK; 1 우, Miryang-si, Sannae-myeon, Mt. Gaji, 27 Apr 2001, Park ES; 8 ð ð JB: Muju-gun, Seolcheon-myeon, Mt. Deogyu, 4 May 1999, Lee JW; 1 우, JN: Jangseong-gun, Bukha-myeon, Yaksu-ri, 252-1, Mt. Naejang, 26 May 2006, Kwon JM; 1 우2 주, SU: Dobong-gu, Dobong-dong, Mt. Dobong, 20 Apr 1983, Jang GS; 1 ð, Seocho-gu, Seocho-dong, Mt. Umyeon, 12 May 1990, Shin JE. Diagnosis. Body 10.9-16.0 mm long, forewing length 10.6$14.6 \mathrm{~mm}$ long. Body black. Palpi, around the compound eye, scutellum, postscutellum, tegula, small subtegular spot, apical parts of fore and mid femur, fore and mid tibia, fore tarsus yellow; ventral part of antenna, teeth of mandible, anterolateral part of mesoscutum, mid and hind dorsal part of femur, mid apical part of tibia, hind tibia, mid and hind tarsus, first metasomal tergite in its posterior one-fifth, 2nd to 8th metasomal tergites yellowish to reddish brown. Clypeus densely punctate with a apicomedian notch. Upper tooth of mandible much wider than lower tooth. Face densely punctate with a projecting small horn between the antennal sockets (Fig. 1E). Distance from the posterior edge of a lateral ocellus to the occipital carina shorter than its maximum diameter. Antenna with 49-56 flagellomeres. Mesosoma entirely densely punctuate (Fig. 1F, G). Notauli very weakly visible. Scutellum strongly convex (Fig. 1F). Propodeum short, not convex and without carinae. Tarsal claws pectinate in its basal three-fourths (Fig. 1D). Nervulus 7.0 times as long as basal vein and 0.8 times ramulus (Fig. 1B).

Host records. Unknown.
Distribution. Korea (new record), China, Japan and Far Eastern Russia.

Subfamily Pimplinae Wesmael, 1845

$1 *$ Genus Theronia Holmgren, 1859

Therionia Holmgren, 1859: 123.

Pseudacoenites Kriechbaumer, 1892: 219. TS: Pseudacoentis moravicus Kriechbaumer.

Poecilopimpla Cameron, 1903: 141. TS: Poecilopimpla lucida Cameron.

Erythrotheronia Cameron, 1905: 134. TS: Erythrotheronia flavolineata Cameron.

Orientotheronia Morley, 1913: 146. TS: Orientotheronia rufescens Morley.

\section{Key to the species of Theronia from Korea}

1. Body reddish brown with black marks. Notaulices shorter, present in approximately apical one-third of the mesoscutum. Lateral carinae of Areola parallel

Theronia atalantae gestator (Thunberg, 1824)

- Body predominantly black. Notaulices longer, present in apical half of the mesoscutum. Lateral carinae of Areola divergent ....... ${ }^{2 *}$ Theronia laevigata nigra Uchida, 1928

\section{Theronia laevigata nigra Uchida, 1928 (Fig. 2)}

Theronia laevigata var. nigra Uchida, 1928: 39.

Material examined. Korea: 3 우 우, CB: Dannyang-gun, Dannyang-eup, Cheondong-ri, Mt. Sobaek, Cheondong valley, 30 Apr-21 May 2007 (MT), Lee JW; 5 우 우, Boeun-gun, Songnisan-myeon, Sanae-ri, Beopjusa, 12-21 Jun 2007 (MT), Lee JW; 1 ð , DG: Nam-gu, Bongdeok-dong, Mt. Ap, 1 May 2004, Lee JW; 1 우, DJ: Dong-gu, Yongun-dong, Daejeon Univ., 18 May-14 Jun 2007 (MT), Lee JW; 1 ð , GB: Cheongdo-gun, Unmun-myeon, Mt. Unmun, 4-30 May 2009 (MT), Lee JW; $2 \sigma^{\nearrow}$ 『, Yeongju-si, Punggi-eup, Jungnyeong, 22 Jun-3 Jul 2009 (MT), Kim CJ; 4 우 우, GG: Gapyeonggun, Cheongpyeong-myeon, Homyeong-ri, Mt. Homyeong, 18 Aug-4 Sep 2009 (MT), Lim JO; 2 우 우, Gwangju-si, Docheok-myeon, Chugok-ri, Mt. Taehwa, 15-25 Jul 2008

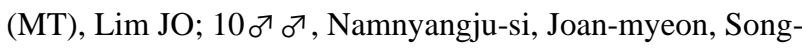
chon-ri, Mt. Ungil, 27 May-10 Jun 2009 (MT), Lim JO; Yangpyeong-gun, Yongmun-myeon, Yeonsu-ri, Doilbong, 27 May-10 Jun 2009 (MT), Lim JO; 1 우, GJ: Nam-gu, Gudong, Gwangju park, 22 Jul 1985, Lim JO; 1 우, GN: Hapcheon-gun, Gaya-myeon, Mt. Gaya, 28 May 1999, Bang SH; 1 우, GW: Wonju-si, Heungeop-myeon, Maeji-ri, Yeonse Univ. Wonju Campus, 2 Sep-20 Oct 2009 (MT), Han HY;

Korean name: ${ }^{1 *}$ 담흑납작맵시벌속(신칭), ${ }^{2 *}$ 검정납작맵시벌 (신칭) 


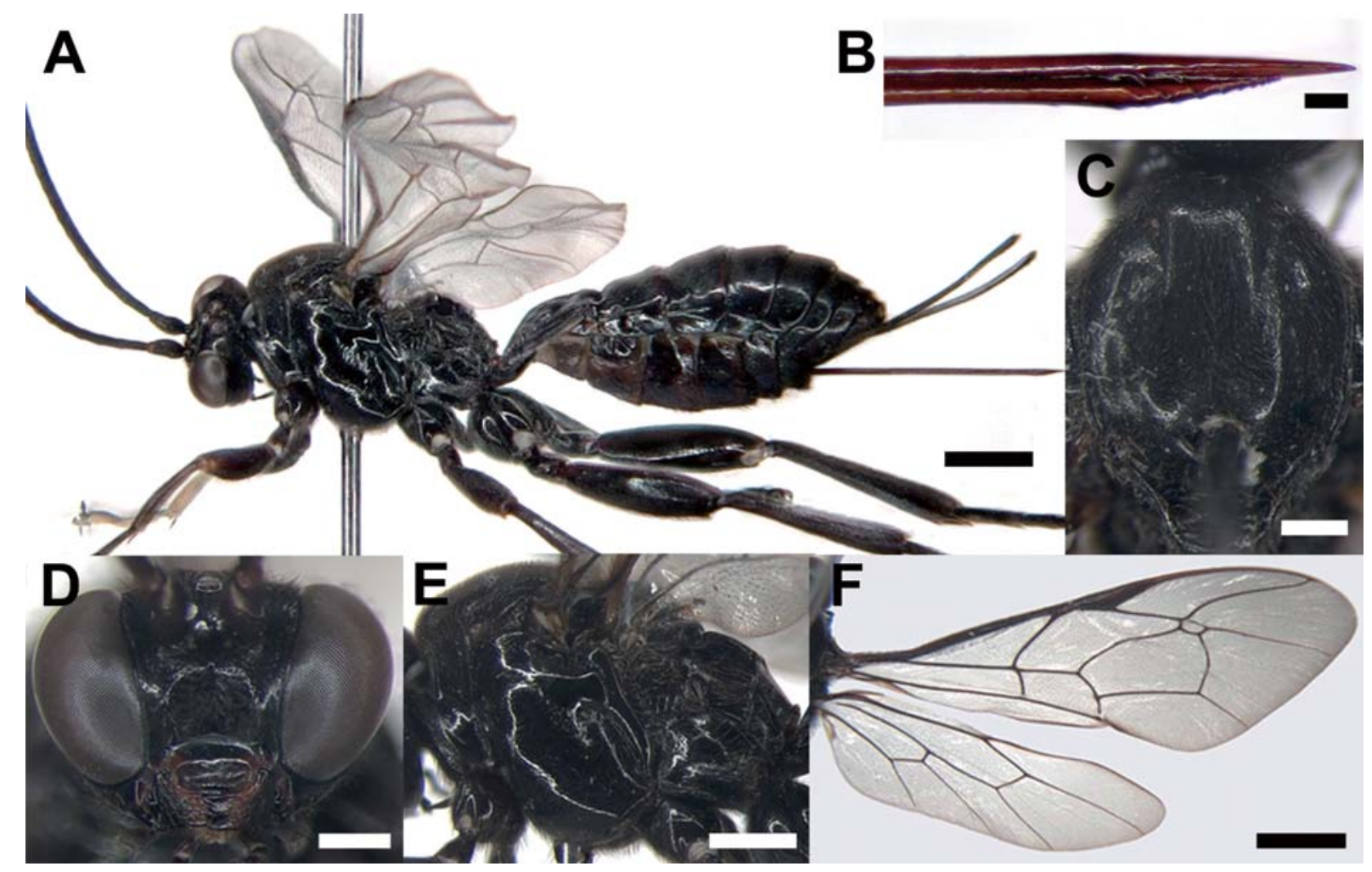

Fig. 2. Theronia laevigata nigra Uchida, 1928, female. A, Lateral habitus; $B$, Ovipositor; C, Mesoscutum in dorsal; $D$, Head in anterior; $E$, Mesosoma in lateral; $F$, Forewing. Scale bars: $A, F=2 \mathrm{~mm}, B=0.1 \mathrm{~mm}, C, D=0.5 \mathrm{~mm}, E=1 \mathrm{~mm}$.

$3 \sigma^{7} \sigma^{7}$, Wonju-si, Panbu-myeon, Seogok-ri, Mt. Baegun, 26 Jun-15 Jul 2007 (MT) Lee JW; 1 우1 $1 \sigma^{7}$, JB: Jeongeup-si, Naejang-dong, Mt. Naejang, Wonjeogam, 25 Jun-15 Jul 2007 (MT), Lee JW; $1 \sigma^{\top}$, JJ: Jeju-si, Donggye-myeon, 14-28 Jul 2005, Sin CH; 1 우, Seogwipo-si, Namwon-eup, Sillyeri, Suakgyo, 28 May 1992, Cha JW; 1 우, JN: Haenam-gun, Samsan-myeon, Pyeonghwal-ri, Mt. Duryun, 3 Oct 2003 (M.N.U.); 1 우, Jangseong-gun, Bukha-myeon, Yaksu-ri, Mt. Naejang, Cheongnyuam, 26 May 2006, Lee JW; 2 우 우, SU: Dobong-gu, Dobong-dong, Mt. Dobong, 6 Jun 1983, Kwon SY; $2 \diamond^{7} \diamond^{\nearrow}$, ditto, 5 Jun 1994, Kim SH; $1 \diamond^{\nearrow}$, US: Ulju-gun, Sangbuk-myeon, Deokhyeon-ri, Mt. Gaji, 19 May 2001, Kang EY; $1 \Im^{\curlyvee}$, ditto, 28 Jun 2001, Park ES.

Diagnosis. Body 7.3-14.5 mm long, forewing length 6.5$12.9 \mathrm{~mm}$ long. Body largely black, except for the following smaller parts are reddish brown. Apical part of mandible (except teeth) and lower part of clypeus brown to reddish brown; dorsal basal margin of mid and hind coxa, ovipositor reddish brown. Clypeus basally slightly convex with irregular several hairs and apically strongly concave with a median notch (Fig. 2D). Face slightly convex medially, with numerous long hairs. Antenna with 36-37 flagellomeres. Mesoscutum with dense short hairs. Scutellum slightly con- vex, apically some punctures with dense long hairs. Propodeal spiracle elongate, 3 times as long as its maximum width. Fore wing vein $2 \mathrm{rs}-\mathrm{m} 0.4$ times as long as 3rs-m. Ovipositor straight, 0.7 times as long as hind tibia, lower valves with nine slanting ridges at the tip (Fig. 2B).

Host records. Malacosoma neustria testacea Motschulsky, Parnassius citrinarius Motschulsky and Parnassius stubbendorfii Ménétriès (Uchida, 1928; Kusigemati, 1988).

Distribution. Korea (new record), Japan and Russia.

\section{ACKNOWLEDGMENTS}

This study was supported by the project of the survey of Korean indigenous species of the National Institute of Biological Resources (NIBR) under the Ministry of Environment, Korea.

\section{REFERENCES}

Cameron P, 1903. Descriptions of new genera and species of Hymenoptera taken by Mr. Robert Shelford at Sarawak, 
Borneo. Journal of the Straits Branch of the Royal Asiatic Society, 39:89-181.

Cameron P, 1905. On the phytophagous and parasitic Hymenoptera collected by Mr. E.Green in Ceylon. Spolia Zeylanica, 3:67-143.

Heinrich GH, 1937. Das Genus Rhynchobanchus und seine europaischen Vertreter. Archiv fur Naturgeschichte, 6:663667.

Holmgren AE, 1859. Conspectus generum Pimplariarum Sueciae. Öfversigt af Kongliga Vetenskaps-Akademiens Förhandlingar, 16:121-132.

Kriechbaumer J, 1892. Xylonomiden und Pimpliden-Studien. Entomologische Nachrichten, 18:211-220.

Kriechbaumer J, 1894. Ichneumonidae novae e fauna Hungarica Musaei Nationalis Hungarici. Termeszetrajzi Fuzetek, 17: 48-60.

Kriechbaumer J, 1896. Entomologia varia. Entomologische Nachrichten, 22:353-372.

Kusigemati K, 1988. Parasitoids of Japanese butterflies. Satsuma, 37:149-160.

Latreille PA, 1802. Histoire naturelle, générale et particulière, des Crustacés et des Insectes. Tome Troisieme, Paris, pp. 318-327.

Meyer NF, 1927. Zur Kenntnis der Tribus Banchini (Familie Ichneumonidae) und einiger neuer Schlupfwespen aus Russland. Konowia, 6:291-311.

Morley C, 1913. The fauna of British India including Ceylon and Burma, Hymenoptera, Vol. 3. Ichneumonidae. British Museum, London, pp. 1-531.

Poda N, 1761. Insecta musei Graecensis. J.B. Dietrich, Graecii, pp. 1-127.

Schulz WA, 1911. Zweihundert alte Hymenopteren. Zoologische
Annalen, Wūrzburg, 4:1-220.

Sheng ML, Li YC, Pang H, 1997. Notes on the genus Rhynchobanchus Kriechbaumer in northeastern China (Hymenoptera: Ichneumonidae). Acta Zootaxonomica Sinica, 22:410413.

Sheng ML, Liu SL, Wang ZC, 1995. A new species of Rhynchobanchus (Hymenoptera: Ichneumonidae) from China. Entomotaxonomia, 17:222-224.

Townes HK, 1969. The genera of Ichneumonidae. Part 1. Memoirs of the American Entomological Institute (Gainesville), 11:1-300.

Townes HK, Momoi S, Townes M, 1965. A catalogue and reclassification of the eastern Palearctic Ichneumonidae. Memoirs of the American Entomological Institute, 5:1-661.

Uchida T, 1928. Dritter Beitrag zur Ichneumoniden-Fauna Japans. Journal of the Faculty of Agriculture, Hokkaido Imperial University, 25:1-115.

Uchida T, 1933. Drei neue japanische echte Schlupfwespen (Hym. Ichneumonidae). Proceedings of the Imperial Academy of Japan, Tokyo, 9:72-75.

Wesmael C, 1845. Tentamen dispositionis methodicae. Ichneumonum Belgii. Nouveaux Memoires de l'Academie Royale des Sciences, des Lettres et Beaux-Arts de Belgique, 18:1238.

Yu DSK, van Achterberg C, Horstmann K, 2012. Taxapad 2012, Ichneumonoidea 2011 [Internet]. Database on flash-drive, Ottawa, ON, Accessed 6 Jul 2012, <http://www.taxapad. com>. 